\title{
Ansiedade e mecanismos de coping utilizados por pacientes cirúrgicos ambulatoriais
}

\author{
ANXIETY AND COPING IN AMBULATORY SURGICAL PATIENTS \\ ANSIEDAD Y MECANISMOS DE COPING UTILIZADOS POR PACIENTES \\ QUIRÚRGICOS DE CONSULTAEXTERNA
}

\section{Lygia Oliveira de Moraes ${ }^{1}$, Aparecida de Cássia Giani Peniche ${ }^{2}$}

1 Aluna de Graduação da Escola de Enfermagem da USP (EEUSP).

lygiamoraes@uol.com.br

2 Professora Doutora do

Departamento de

Enfermagem Médico-

Cirúrgica da EEUSP

ggphe@usp.br

\section{RESUMO}

Os objetivos deste estudo foram identificar o estado de ansiedade e os mecanismos de coping utilizados por pacientes cirúrgicos ambulatoriais no periodo pré-operatório, suas manifestações clínicas e respectivas correlações. Os resultados obtidos identificam que a amostra de 40 pacientes apresentou baixa ansiedade e o mecanismo de coping mais utilizado foi o suporte social, porém, não obtivemos relação estatisticamente significante entre estado de ansiedade e mecanismos de coping e alterações clínicas.

\section{PALAVRAS-CHAVE}

Paciente ambulatorial. Ansiedade.

Mecanismos de "coping".. Cuidados pré-operatórios.

\author{
ABSTRACT \\ The goals of this study \\ were to identify the anxiety \\ state in surgical ambulatory \\ patients in the pre-operative \\ period, the coping mechanisms \\ employed, their clinical \\ manifestation, and to verify \\ the influence of state anxiety \\ during the pre-operative \\ period. The results showed \\ that patients exhibited little \\ anxiety and there was no \\ significantly statistical \\ correlation between pre- \\ operative anxiety state, \\ coping mechanisms and \\ clinical changes.
}

\section{RESUMEN}

Este estudio tuvo como objetivos identificar el estado de ansiedad y los mecanismos de "coping" utilizados por pacientes quirúrgicos de consulta externa en el periodo preoperatorio, sus manifestaciones clinicas y respectivas correlaciones. Los resultados obtenidos identifican que la muestra de 40 pacientes presentó baja ansiedad y el mecanismo de coping más utilizado fue el soporte social, sin embargo, no se obtuvo relación estadísticamente significativa entre el estado de ansiedady mecanismos de "coping" $y$ alteraciones clínicas.

\section{PALABRAS-CLAVE}

Paciente quirúrgico de consulta externa.Ansiedad. Mecanismos de "coping". Cuidados preoperatórios.

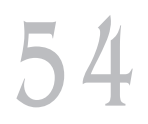

Rev Esc Enferm USP 2003; 37(3): 54-62 


\section{INTRODUÇÃO}

O ressurgimento da cirurgia ambulatorial ocorreu devido ao avanço tecnológico, não só relacionado aos procedimentos cirúrgicos, mas também devido aos novos fármacos utilizados em anestesia ${ }^{(1)}$.

Este avanço, ao beneficiar economicamente hospitais, traz vantagens aos pacientes, uma vez que a intervenção cirúrgica ambulatorial oferece a possibilidade de retorno ao domicílio e possibilita que a recuperação seja com os familiares, sem a necessidade de pernoite no hospital, além do custo ser menor também para o paciente.

Considerando as vantagens da cirurgia ambulatorial, observa-se que estas vêm preencher às necessidades psico-biológicas, psico-emocionais e psicossociais do paciente. Assim, do ponto de vista psicobiológico a cirurgia ambulatorial contribui para evitar ou diminuir o risco de infecção hospitalar, pelo tempo reduzido de permanência do paciente na instituição e, por proporcionar-lhe recuperação mais rápida. Tais vantagens são revertidas em maior satisfação para o paciente. Considerando o aspecto psico-emocional, a realização da cirurgia em ambulatório, colabora para reduzir a ansiedade pré-operatória não só do paciente como também de seus familiares, dada a possibilidade de retorno rápido para o seu ambiente doméstico e à comunidade. Vista sob o aspecto social a cirurgia ambulatorial, além de diminuir o desconforto do paciente gerado pelo afastamento do convívio familiar e de amigos, traz a redução dos custos para o paciente e para a instituição ${ }^{(2-3)}$.

Acreditamos que, independentemente de ser um procedimento realizado em ambulatório com vantagens concretas, a cirurgia e a anestesia continuam sendo consideradas como estímulos desencadeadores de alterações emocionais nos indivíduos quer pela expectativa, pelo medo, pela dor ou até mesmo pelo fato de não ocorrer a internação.

Estas alterações são traduzidas por mudanças hormonais desencadeadas pelo sistema nervoso central e pelo sistema nervoso autônomo simpático e que Selye denominou de estresse. O desencadeamento dessas respostas foi denominado Síndrome de Adaptação Geral.
Estudiosos da temática ${ }^{(4)}$ foram além da resposta biológica do homem ao estímulo, isto é, ampliaram o conceito dado por Selye a esta resposta, incluíram a importância do aparelho cognitivo como mediador da intensidade das respostas aos estímulos, decorrentes das diferentes formas de avaliação feitas pelos indivíduos. Constataram que o ser humano está muito distante de ser apenas um ser biológico, que obedece a um padrão mínimo de respostas. É um ser complexo o qual terá, que conviver com fatores que atuarão na sua estrutura biológica. Isso resultará em demandas psíquicas, as quais serão administradas com graus de competência e resolutividade de acordo com o substrato psíquico de cada um, sua maneira de interpretar os problemas, fazer a avaliação cognitiva, desencadeando alterações hormonais específicas.

Estes mesmos estudiosos ${ }^{(4)}$ definem a avaliação cognitiva como "um processo de categorização de um encontro, com o foco no propósito ou no significado desse encontro". $\mathrm{O}$ significado de encontro para esses autores refere-se à aproximação de dois elementos, sendo que um deles é o sujeito e o outro é de natureza diversa e desencadeador do estímulo.

Nesse processo de categorização, são possíveis dois tipos de avaliação: a primária e a secundária. Esses dois tipos de avaliação feitas pelos indivíduos produzem respostas, desde as mais primitivas até as mais elaboradas, em função das diferentes áreas cerebrais que foram estimuladas.

A avaliação primária ocorre a partir do primeiro contacto do sujeito com o objeto, desencadeando uma apreciação que visa identificar o estímulo. Neste caso, a resposta ou reação classificará o estímulo em relevante ou irrelevante. A partir de então, se for considerado relevante, o homem mobilizar-se-á para a ação.

Outro tipo de avaliação é a secundária onde se torna necessária uma ação para o controle da situação. Envolve uma avaliação sobre o que deve ser feito. É um processo complexo que considera opções de coping ${ }^{(a)}$ disponíveis: expectativa de que uma dada opção de coping irá se realizar, expectativa de se utilizar uma estratégia particular eficaz.

Coping foi definido como estratégias desenvolvidas para enfrentar as situações. Um esforço cognitivo e comportamental, realiza-
Ansiedade e

mecanismos de coping

utilizados por pacientes cirúrgicos ambulatoriais 
Lygia Oliveira de Moraes Aparecida de C. G.Peniche do para dominar, tolerar ou reduzir as demandas externas e internas e o conflito entre elas. Partindo do pressuposto de que as crenças individuais traduzem as idéias e julgamentos sobre a realidade vivenciada e que as normas fundamentam as condutas servindo de guia na compreensão do significado da realidade, é possível supor que ambas tenham influência sobre o coping e, portanto, estabeleçam estreitas relações com o processo de avaliação cognitiva ${ }^{(4)}$.

A forma pela qual uma pessoa usa coping está determinada, em parte, por seus recursos, os quais incluem saúde e energia, crenças existenciais, habilidades de solução de problemas, habilidades sociais, suporte social e recursos materiais, além de ser determinado por variáveis que diminuem o uso dos recursos pessoais.

O coping tem duas funções, segundo a análise desses dois autores, modificar a relação entre a pessoa e o ambiente, controlando ou alterando o problema causador (coping centrado no problema); adequar a resposta emocional ao problema (coping centrado na emoção). Em geral, as formas de coping centradas na emoção ocorrem provavelmente quando já houve uma avaliação de que nada pode ser feito para modificar as condições de dano, ameaça ou desafio ambiental. Formas de coping centrados no problema, por outro lado, são prováveis quando tais condições são avaliadas como fáceis de mudar. Para determinado autor ${ }^{(5)}$ o coping centrado no problema ou na emoção influenciam-se mutuamente em todas as situações estressantes.

Para outro pesquisador ${ }^{(6)}$,adaptação e coping são conceitos distintos. Adaptação é um conceito amplo que abrange desde rotinas, pensamentos e comportamentos automáticos até o próprio coping. Já o coping é um processo utilizado especialmente em situações nas quais o indivíduo não se conduz como normalmente o faria, isto é, implica numa condição de estresse, que demanda a utilização de ações especiais para administrá-lo

Parece haver um consenso de que existe uma correlação estreita entre a percepção sensitiva e cognitiva de uma ameaça, o surgimento da ansiedade e do estresse e as alterações fisiológicas perceptíveis clinicamente $^{(7)}$.
Diante do exposto, defini-se ${ }^{(8)}$ estado de ansiedade como reação emocional transitória percebida pela consciência e caracterizada por sentimentos subjetivos de tensionamento, apreensão, nervosismo e preocupação, intensificando a atividade do sistema nervoso autônomo. Estas respostas incluem alteração da freqüência cardíaca, do padrão respiratório e da pressão arterial, inquietação, estremecimentos, tremores e aumento de sudorese. Afirma-se ${ }^{(8)}$, que a maneira como o indivíduo percebe a ameaça é mais importante do que a própria ameaça. Considera as diferenças individuais na percepção e as reações às situações como traço de ansiedade, isto é, comportamentos individuais que permanecem latentes, até que em uma determinada situação são ativados. Estes comportamentos são influenciados por experiências passadas que levam os indivíduos a reagir de determinada forma.

Para outro autor ${ }^{(9)}$ a ansiedade-estado seria transitória, caracterizada por sentimentos desagradáveis conscientemente percebidos, modificando-se com o tempo e sendo influenciada por fatores externos.

Em pesquisa realizada ${ }^{(10)}$ sobre os pacientes no período pré-operatório imediato temse que todos os pacientes se apresentam estressados e com necessidade de cuidados nesse momento. Esses cuidados, na sua grande maioria, não contemplam o paciente como um ser único. São fornecidos de maneira padronizada, como se todos os indivíduos vivenciassem o mesmo sentimento ou a mesma emoção nessa situação. A participação do paciente nesse processo de avaliação de seu estado de estresse é o que dará subsídios para a elaboração de condutas mais específicas e mais adequadas por parte dos profissionais de enfermagem que prestam os seus cuidados.

No que se refere ao ato cirúrgico, supõese ${ }^{(7)}$ que a antecipação deste evento desencadeará sentimentos e avaliação cognitiva que, influenciados pelas diferenças individuais, resultarão em comportamentos peculiares de ajustes que têm por finalidade enfrentar o estresse e a ansiedade provocada por este evento. Essas avaliações cognitivas serão mais efetivas quanto mais dados da realidade o indivíduo tiver. Várias são as possibilidades de obtenção destes dados, desde experiências passadas até a formulação de dú- 
vidas, questionamentos, hipóteses e associações de idéias relativas à cirurgia e à anestesia.

Para esse mesmo autor ${ }^{(7)}$, ao verificar a correlação entre a ansiedade e a resposta do paciente no período pós-operatório obteve um resultado estatisticamente não significante entre o estado de ansiedade e as respostas do paciente no período pós-operatório, ou seja, apesar da ansiedade desencadear uma reação simpática, esta não foi retratada pela alteração dos parâmetros clínicos. Este resultado pode ter influência de algumas variáveis como a medicação pré-anestésica.

Em outro estudo realizado ${ }^{(11)}$ os autores tiveram como objetivo verificar a influência da ansiedade em pacientes cirúrgicos ambulatoriais com a finalidade de eliminar a variável droga pré-anestésica. Obtiveram resultados semelhantes à pesquisa relatada anteriormente $^{(7)}$, ou seja, não houve correlação estatisticamente significante entre o estado de ansiedade dos pacientes cirúrgicos ambulatoriais e os parâmetros clínicos avaliados no período pós-operatório.

Diante do exposto questionamos o que estaria interferindo no estado de ansiedade e na variação dos parâmetros clínicos compatíveis com a reação simpática desencadeada? Poderiam ser os mecanismos de coping utilizados pelos pacientes neste período? Qual seria a relação dos mesmos com o estado de ansiedade do paciente?

\section{OBJETIVOS}

Identificar o estado de ansiedade em pacientes cirúrgicos ambulatoriais no período pré-operatório e seus parâmetros clínicos; Identificar os mecanismos de coping mais utilizados pelos pacientes cirúrgicos ambulatoriais; Verificar a correlação do estado de ansiedade pré-operatório e mecanismos de coping.

\section{MATERIAL E MÉTODO}

\section{a) população e amostra}

Como critérios para seleção e representatividade da população foram determinados 40 pacientes cirúrgicos ambulatoriais; alfabetizados; faixa etária de 20 a 70 anos; pertencentes ao programa de cirurgias ambulatoriais; submetidos à anestesia local sem sedação; submetidos à intervenção cirúrgica de pequeno e médio porte ${ }^{(12)}$; sem distúrbios orgânicos, fisiológicos, bioquímicos ou psiquiátricos, estado físico 1 (ASA 1) ou com anormalidades fisiológicas, bioquímicas de pequena intensidade, como diabetes mellitus ou hipertensão arterial leve e compensada, estado físico 2 (ASA 2) de acordo com o American Society of Anesthesiology (12-13).

\section{b) instrumentos}

Foram utilizados dois formulários. O formulário 1 é composto por 2 partes. Parte I: dados gerais de identificação como registro geral, idade, sexo, hospita-lização com cirurgias anteriores e intercorrências. Parte II: parâmetros clínicos considerados por diversos autores como constitutivos da avaliação de pacientes cirúrgicos ${ }^{(14-16,18-21)}$, e por estarem associados à atividade nervosa central autonômica e endócrina desencadeadas pela ansiedade ${ }^{(22-23)}$. São eles: freqüência de pulso, pressão arterial, freqüência respiratória, temperatura, reflexos protetores como a tosse e a deglutição, expansibilidade torácica, atividade muscular, sensibilidade cutânea, dor, coloração, nível de consciência, comportamento, sinais vegetativos como vômito, náusea, sialorréia e sudorese.

Os parâmetros utilizados para avaliação clínica no período pré-operatório foram pontuados, com exceção da dor, com base nas variações propostas pelos autores ${ }^{(14-20)}$ e a partir daí criou-se três possibilidades de escores: normal (0), alterado (1) e muito alterado (2).

A avaliação do parâmetro referente à dor, foi baseada na escala analógica ${ }^{(24)}$. É composta por 11 números entre zero a dez (0 a 10). $\mathrm{O}$ zero (0) representa um extremo de dor (ausência) e o dez (10) representa o outro extremo (muita dor). A pontuação dada pelo paciente nessa escala foi classificada nesta pesquisa em normal (0), quando o paciente avaliar sua dor, entre 0 a 3 ; alterada (1), quando o paciente avaliar sua dor entre 4 a 6 e muito alterada (2), quando a pontuação dada for entre 7 a 10 .

Para a categorização destes pacientes considerou-se o parâmetro clínico estava que alterado e o quanto este se encontrava modificado. Por exemplo, o paciente "A" apresentou variação nos seguintes parâmetros clínicos:
Ansiedade e

mecanismos de coping utilizados por pacientes cirúrgicos ambulatoriais 
Lygia Oliveira de Moraes Aparecida de C. G.Peniche temperatura (alterada $=1$ ) e a pressão arterial (muito alterada $=2$ ) e, os demais, dentro da normalidade (normal $=0$ ) resultando no total de 03. Esta soma de escores embasou a correlação feita entre os mecanismos de coping e estado de ansiedade e os parâmetros clínicos.

O segundo formulário, utilizado pelas autoras, foi composto pela escala de estado de ansiedade, parte integrante do Inventário State-Trate Anxiety (IDATE) ${ }^{(25)}$. Essa escala consta de 20 afirmações, em que o sujeito indica a intensidade naquele momento da ansiedade (estado) através de uma pontuação de 1 a 4 . O total da escala varia de 20 a 80 , sendo que os pontos mais altos indicam maior nível de ansiedade.

Em alguns itens desta escala, as pontuações devem ser invertidas, já que o conteúdo das afirmações nela contidas é oposto às afirmações dos demais itens. São eles: 1, 2, 5, 8, 10, $11,15,16,19$ e 20. Para avaliação dos pontos obtidos foi utilizada a categorização já utilizada em outro estudo ${ }^{(26)}$ onde, uma pontuação de 20 a 40 pontos é considerada como baixo estado de ansiedade, de 40 a 60 pontos como médio estado de ansiedade e uma pontuação de 60 a 80 pontos considerada como alto estado de ansiedade. Ao final de cada avaliação, obtevese um total referente ao estado de ansiedade.

Para a identificação dos esforços cognitivos e comportamentais, foi aplicado o inventário de estratégias de coping ${ }^{(4)}$, que consta de 66 itens, englobando pensamentos e ações que as pessoas utilizam para lidar com demandas internas ou externas de um evento estressante específico.

Este inventário foi validado para a língua portuguesa $^{(5)}$ obteve-se 8 fatores, porém consideramos necessária a verificação da consistência interna a partir dos fatores existentes proposto pela autora. Desta verificação um item foi eliminado dos fatores já existentes apresentados a seguir:

Fator 1 - Confronto: 47, 17, 40, 34, 07, 28. Fator 2 - Afastamento:41, 13,44, 21,06, 16, 10. Fator 3 - Autocontrole: 15, 14, 43, 54, 35. Fator 4 - Suporte Social: 42, 45, 08, 31, 18, 22.

Fator 5- Aceitação de Responsabilidade: 51, 09, 52, 29, 48, 25, 62.

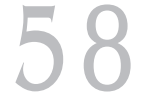

Rev Esc Enferm USP 2003; 37(3): 54-62
Para a obtenção do escore referente ao coping, utilizou-se esta categorização. Zero $(0)=$ não utilizei tal estratégia; um (1) = utilizei pouco; dois(2) = utilizei bastante tal estratégia; três(3) = utilizei em grande quantidade

\section{c) procedimento de coleta de dados}

A coleta dos dados foi realizada no Hospital Universitário da Universidade de São Paulo, mais precisamente na Unidade de Clínica Cirúrgica Ambulatorial onde, após a tramitação e aprovação do projeto pela Comitê de Ética da Instituição. Manteve-se contato com os pacientes cirúrgicos que preenchiam os critérios de seleção da amostra, sendo que os mesmos foram informados sobre a finalidade da pesquisa, da participação voluntária e da garantia do anonimato.

Foram realizadas entrevistas com os pacientes, além da avaliação de seus parâmetros clínicos e aplicação dos questionários, sendo que no dia da cirurgia, aproximadamente 2 horas antes de seu encaminhamento ao Centro Cirúrgico, os pacientes foram questionados quanto aos dados de identificação contidos no formulário 1. A seguir, foi solicitado ao paciente ficar em posição mais confortável possível para serem avaliados os parâmetros clínicos contidos na segunda parte deste mesmo formulário.

Logo em seguida, foram distribuídos os questionários auto-aplicáveis que verificaram respectivamente, o estado de ansiedade do paciente cirúrgico ambulatorial no período pré-operatório e os mecanismos de coping utilizados.

\section{d) procedimento de análise dos dados}

O tratamento estatístico da pesquisa foi realizado primeiramente utilizando o teste de alfa de Cronbach onde se verificou a consistência interna do instrumento de coping através de um índice de confiabilidade que oscila entre valores de 0,00 e 1,00 . Neste estudo, considerou-se como confiabilidade um alfa de Cronbach de 0,50 . $O$ fator 3 - autocontrole apresentou um alfa abaixo de 0,50 , porém com a retirada do item 35 foi possível aumentar este valor não sendo necessária a retirada deste fator no estudo.

Para as seguintes análises do estudo foram realizados os cálculos de médias-padrão, considerados os valores de mínimos e máximos, desvios-padrão e medianas. 
As relações entre as variáveis estado de ansiedade e estratégias de enfrentamento foram obtidas, aplicando-se os testes não paramétricos de prova de "U" de MannWhitney e o coeficiente de relação de Spearman.

\section{RESULTADOS E DISCUSSÃO}

A amostra deste estudo foi constituída por 40 pacientes sendo deste total, 21 pacientes do sexo masculino (52\%) e 19 pacientes do sexo feminino ( $48 \%$ ).

Em relação à faixa etária encontramos 09 pacientes com idade entre 20 e 30 anos (22\%), 07 pacientes com idade entre 31 e 40 anos (18\%), 05 pacientes com idade entre 41 e 50 anos (13\%), 10 pacientes com idade entre 51 a 60 anos $(24 \%)$ e 09 pacientes com idade entre 61 e 70 anos (23\%).

Com relação à experiência cirúrgica pregressa, 12 pacientes não haviam realizado cirurgias anteriores $(30 \%)$ e 28 pacientes já realizaram $(70 \%)$. Este fato possivelmente trará para a avaliação da situação atual, sentimentos e enfrentamentos impregnados das situações anteriores e expressos no estado emocional atual assim como nos parâmetros clínicos avaliados ${ }^{(7)}$.

Dos 28 pacientes que já experienciaram cirurgias anteriores, $19(68 \%)$ não relataram a presença de intercorrências em suas cirurgias (68\%).

Mesmo tendo um número elevado de pacientes sem intercorrência no procedimento anestésico cirúrgico, não se pode desconsiderar o fato dos 19 pacientes (68\%) terem suas avaliações e comportamentos marcados pelas lembranças e que essas podem estar contribuindo atualmente na construção de mecanismos que os auxiliem nesta experiência.

Com relação ao estado de ansiedade encontramos, 26 pacientes com baixo estado de ansiedade (64\%), 12 pacientes com médio estado de ansiedade (33\%) e 2 pacientes com alto estado de ansiedade (3\%). Estes dados caracterizam uma amostra composta por pacientes com baixo estado de ansiedade (64\%). $\mathrm{Na}$ maioria dos estudos realizados tem-se encontrado pacientes com baixo estado de ansiedade o que nos leva a questionar sobre a veracidade das informações obtidas com o questionário. Acredita-se que existência da ansiedade inconsciente, apesar de estar presente, não pode ser detectada por vários testes e levar a uma falsa avaliação ${ }^{(8)}$.
Em um serviço de cirurgia, ${ }^{(27)}$ constatou-se que $97 \%$ dos pacientes experimentaram algum grau de ansiedade. Quando indagados quais aspectos aumentaram a ansiedade sentida, os sujeitos apontaram, com maior freqüência, o procedimento anestésico, a espera na unidade pelo procedimento cirúrgico e a possibilidade de dor e desconforto; quando questionados quais os aspectos que minimizam a ansiedade, os pacientes responderam com maior incidência: as informações recebidas da enfermeira, o ambiente da unidade e as informações recebidas do anestesiologista e a permanência destes ao seu lado durante o procedimento.

É possível supor com isso que, o fato das cirurgias serem ambulatoriais, sem anestesia geral e em um instituição, onde a orientação do paciente é priorizada tenha contribuído para os resultados aqui obtidos.

Mesmo diante dessa situação específica, foi possível observar, independentemente do grau de ansiedade, alterações dos valores dos parâmetros clínicos basais ${ }^{(7)}$.

Do total de pacientes estudados, encontramos $10(25 \%)$ pacientes sem variação de parâmetros clínicos; 12 (30\%) pacientes com variação de um (1) ponto; 10 (25\%) pacientes com variação de dois (2) pontos; 4 (10\%) pacientes com variação de três (3) pontos; 2 (5\%) pacientes com variação de quatro (4) pontos e $2(5 \%)$ com variação de cinco (5) pontos no formulário de parâmetros clínicos.

Para determinado autor ${ }^{(28)}$ nem todos os indivíduos reagem ao estresse da mesma forma, ou seja, os órgãos e sistemas que reagem ao estresse também variam de pessoa para pessoa. Enquanto para uns a manifestação se dá no trato gastrointestinal, para outros pode ser no sistema cardiovascular ou músculoesquelético. $\mathrm{O}$ resultado imediato do estresse transitório ou crônico é a adaptação que pode ser uma resposta patológica ou fisiológica de diferente intensidade, duração ou qualidade.

Com relação ao agrupamento do mecanismo de coping mais utilizado pelos pacientes cirúrgicos ambulatoriais, em média $(1,57)$, foi o de suporte social, seguido dos agrupamentos de resolução de problemas $(1,44) \mathrm{e}$ reavaliação positiva $(1,41)$.

Acreditamos que os pacientes da amostra contaram com o apoio de parentes e amigos, assim como, com a procura de mais in-
Ansiedade e

mecanismos de coping utilizados por pacientes cirúrgicos ambulatoriais 
Lygia Oliveira de Moraes Aparecida de C. G.Peniche formações sobre a cirurgia encontrando-os na própria filosofia institucional que proporciona ao paciente o acesso para o esclarecimento de suas dúvidas e incertezas.

O agrupamento resolução de problemas pertence à classificação de enfrentamento centrado no problema e pode ajudar o indivíduo a reduzir a emoção eliminando as demandas ambientais, por meio da busca de informações e ações de solução de problemas. Já o agrupamento de reavaliação positiva está centrado na emoção e ao desenvolvimento de planos de ação para minimizar as emoções negativas, por meio da regulação afetiva ou da descarga emocional. O indivíduo tenta atenuar o desconforto emocional, dando uma interpretação realista ou distorcida do fato.

Dentre as muitas estratégias de enfrentamento existentes, o que determina sua eleição é a natureza do estressor, as circunstâncias em que ele se reproduz, a história prévia de confronto e o próprio estilo que caracteriza o sujeito, ou seja, como ele enfrentou uma situação passada. Há dois tipos básicos de indivíduos, aqueles que têm tendência evitadora ou minimizadora e aqueles com estilo afrontativo ou vigilante ${ }^{(29)}$.

Em relação ao estado de ansiedade ,relacionado à distribuição dos pacientes quanto ao sexo, temos que no sexo feminino a média foi de 38,8 pontos caracterizando-se assim como uma amostra de baixo estado de ansiedade, assim como nos pacientes do sexo masculino, porém com média de 33,8 pontos.

Em outra pesquisa realizada ${ }^{(11)}$ as mulheres também foram as que apresentaram maior média de estado de ansiedade no período pré-operatório. Esta variação, embora decimal e estatisticamente não significante, aponta para a uma resposta positiva quando expostas à cirurgia.

Com relação à variação de parâmetros clínicos, as mulheres apresentaram média de 2,0 pontos, ou seja, no mínimo 2 parâmetros clínicos alterados e os homens obtiveram média de 1,1 . Os dados nos mostram que os homens estavam clinicamente mais estáveis e possivelmente menos ansiosos que as mulheres e assim não apresentaram estado de ansiedade alto, nem variações clínicas significantes, ou ainda que, como ressalta determinado autor ${ }^{(30)}$ que os indivíduos utilizando-se das estratégias de coping, conseguiram resolver os conflitos sem solicitar do organismo alterações significativas dos parâmetros clínicos.
Apesar do teste estatístico realizado mostrar que entre o grupo dos homens e o das mulheres não foram encontradas diferenças significantes quanto a utilização dos mecanismos de coping, ao analisarmos as médias referentes ao agrupamento de coping utilizado, encontramos que os homens utilizaram mais o suporte social, enquanto que para as mulheres o mais utilizado foi o da reavaliação positiva. Em relação à idade, encontramos uma distribuição do estado de ansiedade onde, a faixa etária com maior média de estado de ansiedade foi a de 20-30 anos com 41,6 pontos, seguida pela faixa de 41-50 anos com média de 36,4 pontos. A faixa etária menos ansiosa foi de 61-70 anos onde a média de estado de ansiedade indicou 31,7 pontos.

Em estudo ${ }^{(30)}$ sobre a ansiedade, depressão e coping em idosos, obteve-se resultados que evidenciaram a presença de médio a baixos níveis de ansiedade nesses pacientes.

Em relação à variação de parâmetros clínicos, temos a maior variação na faixa etária de 41-50 anos com média de 2,4 pontos. A faixa etária com menor média foi a de 31-40 anos com 0,8 pontos. As faixas etárias de 20-30 anos, 51-60 anos e 61-70 anos obtiveram médias de 1,4 nas duas primeiras e 1,8 na última.

Nos pacientes com cirurgias anteriores, a média de estado de ansiedade é de 34 pontos enquanto que a média daqueles sem experiência é de 41,2 pontos, estes dados nos mostram que, os pacientes, com experiência cirúrgica anterior, responderam com menor ansiedade do que aqueles sem experiência. Talvez, por não terem nenhuma referência simbólica relativa à vivência de um evento cirúrgico, respondem com maior ansiedade a este momento.

Estes resultados encontrados, corroboram com aqueles referentes ao estado de ansiedade no período pré-operatório ${ }^{(11)}$ onde também, existia um estado maior de ansiedade para os pacientes sem experiências anteriores. Embora os pacientes com menor ansiedade tenham sido aqueles com experiências prévia, estes apresentaram uma variação maior dos parâmetros clínicos (média de 1,6 pontos) enquanto os pacientes sem experiência anterior obtiveram uma média de 1,3 pontos.

Quanto aos mecanismos de coping utilizados, o teste estatístico realizado mostra que entre os dois grupos (com ou sem experiência cirúrgica anterior) não foram encontradas diferenças significantes, Esse resultado é reforçado ao constatarmos que o agrupamen- 
to mais utilizado tanto pelos pacientes com ou sem experiências anteriores foi o de suporte social e o agrupamento menos utilizado pelos dois grupos foi o de confronto.

Quanto ao estado de ansiedade dos pacientes em relação à existência de intercorrências temos que a média dos pacientes com intercorrências é de 37 pontos enquanto dos sem intercorrências é de 32,6 pontos. Estes resultados mostram que os pacientes, que em suas experiências anteriores, apresentaram algum tipo de problema, responderam com uma média de ansiedade, maior do que aqueles que não apresentaram problemas.

Encontramos dados correspondentes em outra pesquisa ${ }^{(11)}$ realizada com pacientes cirúrgicos ambulatoriais onde também os pacientes com intercorrências apresentaram maior estado de ansiedade pré-operatória.

Em relação à variação dos parâmetros clínicos temos que o grupo com maior média na variação foi o dos pacientes com intercor-rência com 2,2 pontos de média, enquanto os pacientes sem intercorrência apresentaram média de 1,3 pontos.

Quanto aos mecanismos de coping utilizados, o teste estatístico realizado nos mostra que entre os dois grupos (com ou sem intercorrência) não foram encontradas diferenças significantes, porém ao analisarmos qual o agrupamento mais utilizado foi o de suporte social e o menos utilizado foi o de confronto.

A tendência em escolher uma determinada estratégia de coping depende do repertório individual (muitas estratégias podem ser úteis para uma mesma situação) e de experiências tipicamente reforçadas.

Como a amostra não apresentou uma distribuição normal dos mecanismos de coping entre os grupos de ansiedade baixa e média considerou-se necessário aplicar o teste de MannWhitney com a finalidade de comparar os dois grupos. Observou-se diferença significativas entre os dois grupos de ansiedade (pacientes com baixa e média ansiedade) no que se refere à utilização dos mecanismos de coping, ou seja, o fator 1 -confronto $(p=0,003)$ foi mais utilizado por aqueles pacientes com ansiedade média, seguido pelo fator 4 -suporte social $(\mathrm{p}=0,001)$, $\mathrm{o}$ fator 5 -aceitação de responsabilidade $(\mathrm{p}=0,071)$ e o 6 - fuga e esquiva ( $p=0,032)$.

Os pacientes com ansiedade média apresentaram valores significantemente maiores nas estratégias de enfrentamento: confronto, suporte social, aceitação de responsabilidade e fuga e esquiva.
Continuando a análise entre as variáveis de estado de ansiedade e mecanismos de coping, foi realizado o teste de correlação de Spearman, onde não foram encontradas correlações estatisticamente significantes apesar de utilizarem mais de uma estratégia que de outra.

Mesmo não havendo uma correlação estatisticamente significante encontramos uma tendência para tal nos mecanismos de coping relacionados à fuga e esquiva $(0,0565)$. Entendemos assim que, a medida em que o estado de ansiedade aumenta, existe uma tendência de utilização dos mecanismos de fuga e esquiva.

Como já citado na introdução, em geral as formas de coping centradas na emoção são passíveis de ocorrer, quando já houve uma avaliação de que nada pode ser feito para modificar as condições de dano, ameaça ou desafio ambiental, o que acreditamos seja a situação dos pacientes cirúrgicos aqui estudados, pois sabem da necessidade da realização da cirurgia e portanto não podem mudar a situação ${ }^{(4)}$

Em pesquisa realizada ${ }^{(31)}$, considera-se que se o coping for eficiente atenua o estresse. Porém quando as estratégias de coping não conseguem resolver os conflitos, as mudanças fisiológicas passam a atuar em função do coping. As estratégias de coping podem ser avaliadas quanto aos resultados produzidos. Quando conseguem controlar os eventos estressantes, adaptando os indivíduos às mudanças, considera-se que as estratégias foram eficientes. Se o indivíduo chega a uma situação de exaustão, essas estratégias não foram suficientes. Pode haver também estratégias de coping deficientes ou ainda paliativas, onde depois de algum tempo o indivíduo retorna à situação estressante. $\mathrm{O}$ indivíduo terá maior possibilidade de dominar a situação estressora quanto mais resolutivas forem as estratégias de coping de que dispõe.

\section{CONCLUSÕES}

A maioria dos pacientes cirúrgicos ambulatoriais no período pré-operatório apresentou baixo estado de ansiedade e com variação normal dos parâmetros clínicos, ou seja, compatíveis com o estado emocional apresentado.

Os mecanismos de coping mais utilizados foram o de suporte social, seguido pelo de resolução de problemas e reavaliação positiva.

Apesar de não existir uma correlação estatisticamente significante entre ansiedade e mecanismos de coping utilizados, encontramos uma tendência na utilização do mecanismo de fuga e esquiva.
Ansiedade e

mecanismos de coping

utilizados por pacientes cirúrgicos ambulatoriais 


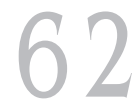

Rev Esc Enferm USP 2003; 37(3): 54-62.

\section{REFERÊNCIAS}

(1) Watson S. Cirurgia ambulatorial. In: Meeker MH, Roth RJC, editores. Alexandre cuidados de enfermagem ao paciente cirúrgico. 10 ${ }^{\mathrm{a}}$ ed. Rio de Janeiro: Guanabara Koogan; 1997. p. 134-68.

(2) Brunner LS, Suddarth DS. Tratado de enfermagem médico-cirúrgica. Rio de Janeiro: Interamericana; 1990.

(3) Smeltzer SC, Bare BG. Tratado de enfermagem médico-cirúrgica. Rio de Janeiro: Guanabara Koogan; 1994.

(4) Lazarus RS, Folkman S. Cognitive appraisal processes. In: Stress appraisal and coping. New York: Springer Publishing Company; 1984. p.22-54

(5) Savoia MG, Santana, PR, Mejias, NP. Adaptação do inventário estratégias de coping de Fokman e Lazarus para o português. Psicol USP 1996; 7 (1/2):183-201.

(6) Portnoi AG. Dor, stress e coping: grupos operativos em doentes com síndromes de fibromialgia. [tese] São Paulo (SP): Instituto de Psicologia da USP; 1999.

(7) Peniche ACG. A influência da ansiedade na resposta do paciente no período pós-operatório imediato. [tese] São Paulo (SP): Escola de Enfermagem da USP; 1998.

(8) Spielberger C. Tensão e ansiedade. Nova Deli: Harper \& Row do Brasil; 1979.

(9) Andreatini R, Leite JR. IDATE - traço: adaptação para avaliação da ansiedade durante sete dias. J Bras Psiq 1994; 43(5): 259-65.

(10) Costa ALS. Análise do stress nas situações de vida diária e do pré-operatório imediato de pacientes cirúrgicos urológicos. [dissertação] São Paulo(SP): Escola de Enfermagem da USP; 1997.

(11) Peniche ACG, Moraes LO. Ansiedade no paciente cirúrgico ambulatorial. São Paulo, 2001. (Relatório final de pesquisa apresentado CNPq-PIBIC).

(12) Faintuch J, Faintuch JJ. Observação clínica pré-operatória. In: Faintuch J, Machado MCC, Raia AA. Manual de pré e pós-operatório. São Paulo: Manole; 1978. p. 03-13.

(13) Hoffer JL. Anestesia. In: Meeker MH, Roth RJC. Alexandre cuidados de enfermagem ao paciente cirúrgico. $10^{\mathrm{a}}$ ed. Rio de Janeiro: Guanabara Koogan; 1997. p. 134-68.

(14) Aldrete JA, Kroulik DA. Postanesthetic recovery score. Curr Rev Anesth 1970; 49(6): 924-33.

(15) Posso MBS. Avaliação das condições dos pacientes na sala de recuperação pós-anestésica. Rev Esc Enferm USP 1975; 9(3): 9-23.
(16) Beland I, Passos J. Enfermagem clínica: aspectos fisiológicos e psicológicos. $3^{\mathrm{a}}$ ed. São Paulo: EPU; 1978. cap.7, p.319-79.

(17) Bevilacqua RG. Alterações endócrinas e metabólicas no trauma. In: Allgower M, Bevilacqua RG, editores. Manual de cirurgia. São Paulo: EPU; 1981. p.21-33.

(18) Castaños CC. Índices de recuperação. Rev Bras Anestesiol 1982; 32(6): 441-2.

(19) Schneider M. Meeting the criterio for discharge. Curr Rev Recov Room Nurses 1982; 4(6): 41-8.

(20) Griffin DN. A tool to develop standarts of quality care in Pacus. J Post Anesth Nurs 1989; v.4: 99-102.

(21) DeLucia R, Planeta CS. Ansiolíticos. In: Valle LBS, editor. Farmacologia integrada: fundamentos farmacológicos da terapêutica. São Paulo: Ateneu; 1991. p. 87-91.

(22) Mc Gaffigan PA, Christoph SB. Assessment and monitoring of the post anesthesia patient. In: Drain CB, editor. The post anesthesia care unit: a critical care approach to post anesthesia nursing. Philadelphia: WB Saunders Company 1994; p.261-88.

(23) Jensen MP, Karoly P, Brauer S. The measurement of clinical pain intensity: a comparison of six methods. Pain 1986; 27:117-26.

(24) Spielberger C, Gorsuch RL, Lushene RE. Inventário de ansiedade traço-estado. Rio de Janeiro: CEPA; 1979.

(25) Chaves EC. Stress e trabalho do enfermeiro: a influência de características individuais no ajustamento e tolerância ao turno noturno. [tese] São Paulo (SP): Instituto de Psicologia da USP; 1994.

(26) Mitchell M. Psycological preperation for patient undergoing day surgery. Ambul Surg 2001; 8(1):19-29.

(27) Handly M, Nelff P. Anxiety and panic attacks. New York: Fawcet Crest 1995; p.21.

(28) Kapplan HI, Sadock. Compêndio de psiquiatria dinâmica. $3^{a}$ ed Porto Alegre: Artes Médicas 1984; p.413-8.

(29) Galdino JMS. Ansiedade, depressão e coping em idosos. [dissertação] São Paulo (SP): Escola de Enfermagem da USP; 2000.

(30) Farah OG. Stress e coping no estudante de Graduação em Enfermagem: investigação e atuação. [tese] São Paulo (SP): Escola de Enfermagem da USP; 2001. 